\title{
Narrative review of optimal prognostic radiological tools using computed tomography for T1N0-staged non-small cell lung cancer
}

\author{
Takeo Nakada, Hiroaki Kuroda \\ Department of Thoracic Surgery, Aichi Cancer Center Hospital, Nagoya, Japan \\ Contributions: (I) Conception and design: Both authors; (II) Administrative support: T Nakada; (III) Provision of study materials or patients: T \\ Nakada; (IV) Collection and assembly of data: T Nakada; (V) Data analysis and interpretation: T Nakada; (VI) Manuscript writing: Both authors; (VII) \\ Final approval of manuscript: Both authors. \\ Correspondence to: Hiroaki Kuroda. Department of Thoracic Surgery, Aichi Cancer Center Hospital, 1-1 Kanokoden, Chikusa-ku, Nagoya 464-8681, \\ Japan. Email: h-kuroda@aichi-cc.jp.
}

\begin{abstract}
Various radiological tools can predict the prognosis of non-small cell lung cancer (NSCLC). In this study, we evaluated the prognostic effect of different radiological tools such as whole tumor size (WTS), consolidation size (CS), consolidation tumor ratio (CTR), tumor disappearance ratio (TDR), mediastinal diameter (MD), and ground glass opacity (GGO) using high-resolution computed tomography (HRCT). We reviewed recent retrospective studies on the predictive effect of these radiological tools on disease-free survival (DFS) and overall survival (OS) in patients with T1N0-staged NSCLC. We searched PubMed and the British Library databases for the English literature published from January 2010 to December 2020 and generated a total of 32 publications (NSCLC, $n=16$; adenocarcinoma, $n=16$ ). The TNM classification version 7 was used in 18 studies, and version 8 in 14 studies. The evaluated radiological parameters were WTS, CS including T category, CTR, TDR, MD, presence of GGO, GGO ratio, and pure GGO. This review suggested that $\mathrm{CS}, \mathrm{MD}$, and the presence of GGO are optimal prognostic radiological tools for cT1N0-Staged NSCLC. CTR or TDR for part solid nodules (PSNs) is not a well-accepted prognostic factor. Further investigations are required to differentiate between benign scars and malignant components on HRCT and evaluate the prognosis of PSNs $(1<\mathrm{CS} \leq 2 \mathrm{~cm})$ with large WTS in the future.
\end{abstract}

Keywords: Non-small cell lung cancer (NSCLC); computed tomography; prognosis; tumor size; ground glass opacity (GGO)

Submitted Nov 24, 2020. Accepted for publication Apr 02, 2021.

doi: $10.21037 /$ jtd-20-3380

View this article at: http://dx.doi.org/10.21037/jtd-20-3380

\section{Introduction}

Lung cancer is the most common cancer worldwide and a leading cause of cancer-related mortality. The rapid adoption of computed tomography (CT) is enhancing early detection of lung cancer. The $8^{\text {th }}$ tumor-node-metastasis (TNM) classification for non-small cell lung cancer (NSCLC) has been embraced worldwide. High-resolution computed tomography (HRCT) is crucial for defining the clinical T category of NSCLC and measuring radiological markers such as whole tumor size (WTS), consolidation size (CS), consolidation tumor ratio (CTR), tumor disappearance ratio (TDR), and mediastinal diameter (MD).

In the $8^{\text {th }}$ TNM classification, the clinical T category is assigned to remnant WTS without ground glass opacity (GGO) on HRCT. In 2019, Kim et al. reported that CTR and TDR were not independent long-term prognostic factors for NSCLC compared with the clinical T category (1). However, Japanese oncological trials (JCOG 0802/0804) have emphasized the malignant behavior of 
NSCLC, hence underscoring the importance of CTR in predicting the outcomes of the tumor $(2,3)$. Therefore, in this study, we evaluated the current literature and published data of our institute regarding prognostic radiological tools using HRCT, including positron emission tomography/ computed tomography (PET/CT), for stage I NSCLC. We reviewed recent retrospective studies on the predictive ability of CT-based radiological tools (CS, CTR, TDR, MD, GGO, and solid tumor) on disease-free survival (DFS) and overall survival (OS) in patients with T1N0-staged NSCLC. Regarding GGO, parameters were separated into GGO ratio and presence of GGO (part solid tumor compared with pure solid tumor).

We present the following article in accordance with the Narrative review reporting checklist (available at http:// dx.doi.org/10.21037/jtd-20-3380).

\section{Methods}

We searched the PubMed and British Library databases for relevant literature published from January 2010 to December 2020. We searched for English literature using the following string: non-small cell lung cancer AND computed tomography AND prognosis AND (tumor size OR consolidation size OR solid component size OR consolidation tumor ratio $\mathrm{OR}$ tumor disappearance ratio OR mediastinal diameter OR ground glass opacity OR T-category OR part solid). We checked the details of each publication to avoid duplication. The differences were resolved by consensus.

\section{Inclusion criteria}

Our inclusion criteria were (I) studies that focused on the application of CT features for clinical or pathological stage I NSCLC, (II) studies that evaluated the use of CT to predict DFS or OS for stage I NSCLC, (III) studies published from 2010 to 2020, (IV) studies published in English, and (V) studies available in full-text.

\section{Exclusion criteria}

The exclusion criteria were (I) studies that did not meet the purpose of this review, (II) studies that evaluated other outcomes except DFS or OS, other radiological tools, stage II-IV of NSCLC, small cell lung cancer, radiation therapy, chemotherapy, and multiple nodules, and (III) case series.

\section{Data extraction}

From each included study, we collected the following data: name of the first author, publication year, number of patients, age, gender, TNM version, tumor size according to TNM version, pathological type (NSCLC and adenocarcinoma), prognostic outcomes, main radiological tools, and available parameters for better prognosis.

\section{Results}

The literature search yielded 32 publications from PubMed and British Library databases (Figure 1). Of these, 16 publications focused on NSCLC, while 16 focused on adenocarcinoma (Figure 2). The study characteristics are shown in Table 1. All studies were retrospective in nature. The TNM version 7 was used in 18 studies, and version 8 in 14 studies. For adenocarcinoma, more studies were published from 2018 than in prior years. In some instances, five authors have reported two or more studies. The median number of patients was 345.5 (range, 71-2,010), and the proportion of males was $45.1 \%$ (median, range, $28.5-$ $77.6 \%$ ). The median or mean age range was $54-71$ years. The evaluated radiological parameters were 11 WTS, 14 CS, including T category, 13 CTR, 5 TDR, 2 MD, 8 GGO, 2 GGO ratio, and 1 pure GGO.

\section{Published reports}

\section{NSCLC}

NSCLC was evaluated in 16 studies. The TNM version 7 was used in 11 studies, while version 8 was used in 5 studies. The median number of patients was 294 (range, $71-2,010)$, and the median percentage of males was $50.8 \%$ (range, 34.7-68.6\%). The median or mean age range was 54-71 years. The evaluated radiological parameters were 3 WTS, 6 CS, including T category, 6 CTR, 2 TDR, 1 MD, 5 presence of GGO, 1 GGO ratio, and 1 pure GGO. After excluding pure GGO or presence of GGO in univariate analysis, the final prognostic parameters for NSCLS were 5 CS including T category, 2 CTR, $1 \mathrm{MD}, 1$ TDR, 4 presence of GGO, and 1 GGO ratio. For NSCLC, CS, including $\mathrm{T}$ category and presence of GGO were indicators of better prognosis.

\section{NSCLC in clinical stage T1N0M0}

Fourteen publications focused on NSCLC in clinical stage T1N0M0. The TNM 7 was used in 10 studies, while TNM 


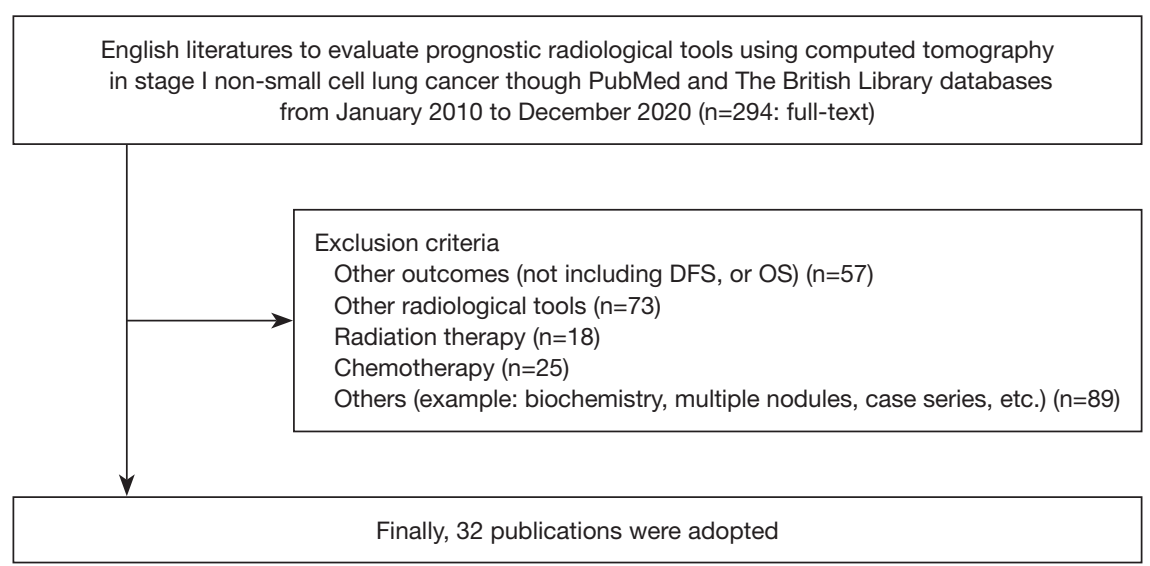

Figure 1 Flow diagram. Inclusion and exclusion criteria for articles used in the review. DFS, disease-free survival; OS, overall survival.

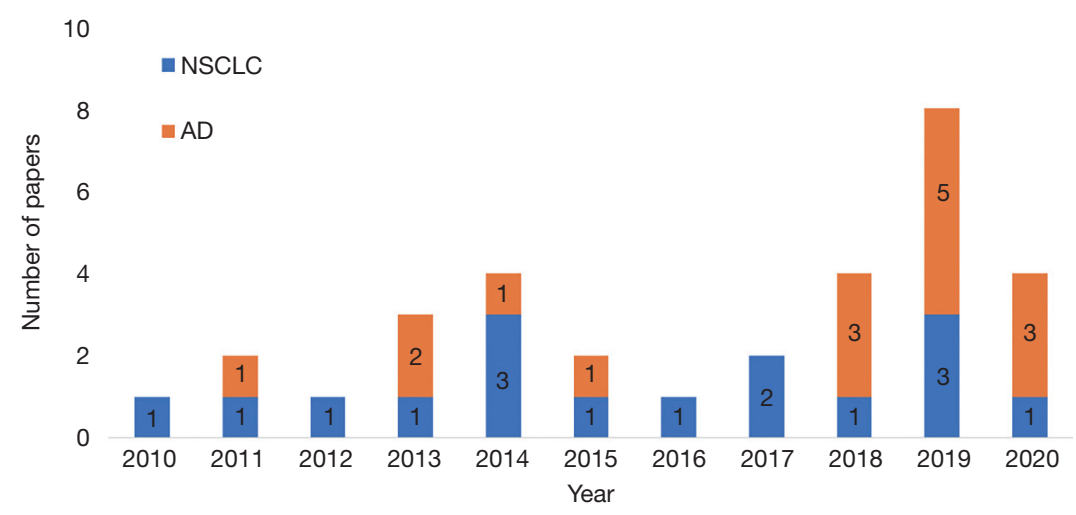

Figure 2 The studies to evaluate prognostic radiological parameters using computed tomography in NSCLC and AD. NSCLC, non-small cell lung cancer; AD, adenocarcinoma.

8 was used in 4 studies. After excluding pure GGO, the available parameters for better prognosis were 1 WTS (only univariate analysis), 4 CS including T category, 3 of CTR (1 of 4 was only univariate analysis), 2 TDR, 3 presence of GGO, and 1 GGO ratio. Finally, CS, including T category, CTR, and presence of GGO were indicators of better prognosis.

Based on TNM 7, Yano evaluated 1,737 patients with stage cT1N0 NSCLC who underwent limited resection (14). CTR $>0.25$, was an independent predictor of recurrence [hazard ratio $(\mathrm{HR})=2.62 ; \mathrm{P}<0.05]$. Using TNM 7, Koike evaluated 328 patients with stage cT1N0 NSCLC who underwent sublober resection (8). They evaluated the WTS and CTR for cancer-specific survival and local recurrence. In the univariate analysis, WTS and CTR were statistically independent predictors, but this prognostic effect was not observed in the multivariate analysis.
Hattori evaluated 497 patients $(\leq 3 \mathrm{~cm})$ using TNM 7 (20). They evaluated GGO component, WTS $(\leq 20 \mathrm{~mm}$ or $21-30 \mathrm{~mm})$, and CS $(\leq 20 \mathrm{~mm}$ or $21-30 \mathrm{~mm})$ for 5 -year OS between part-solid $(0.5 \leq \mathrm{CTR}<1.0)$ and pure-solid $(\mathrm{CTR}=1.0)$ groups. The 5 -year OS was significantly different between pure solid and part-solid tumors $(82.7 \%$ vs. $95.3 \%, \mathrm{P}<0.0001)$. For OS, the HR of the GGO component was 3.325 . Furthermore, it was identical despite the maximum tumor size $(\leq 20 \mathrm{~mm}, 96.6 \% ; 21$ to $30 \mathrm{~mm}, 94.9 \% ; \mathrm{P}=0.4810$ ) or the solid component size ( $\leq 20 \mathrm{~mm}, 96.0 \% ; 21$ to $30 \mathrm{~mm}, 93.8 \% ; \mathrm{P}=0.6119)$. The same author also evaluated 71 patients $(\leq 1 \mathrm{~cm})$ under TNM 7 and 328 patients $(\leq 3 \mathrm{~cm})$ under TNM 8 to analyze the GGO component for DFS and OS $(15,19)$. In both reports, the authors suggested that the GGO component was an indicator of better prognosis. Takenaka evaluated 255 patients $(\leq 3 \mathrm{~cm})$ using TNM 7 (17). They 
Table 1 Characteristics of prognostic radiological tools using computed tomography in T1N0-Staged non-small cell lung cancer

\begin{tabular}{|c|c|c|c|c|c|c|c|c|c|c|c|}
\hline $\begin{array}{l}\text { First author } \\
\text { (reference) }\end{array}$ & Year & $\begin{array}{l}\text { No. of } \\
\text { patients }\end{array}$ & Age (year) & Gender (male) & $\begin{array}{l}\text { Stage (tumor size } \\
\text { according to no of edition) }\end{array}$ & $\begin{array}{l}\text { TNM } \\
\text { version }\end{array}$ & Pathology & Surgery & Prognosis & Evaluated radiological tools & Available parameters for better prognosis \\
\hline Inoue M (4) & 2010 & 118 & 65 (median) & $41(34.7 \%)$ & Clinical IA $(\leq 2 \mathrm{~cm})$ & 7 & NSCLC & $\mathrm{W}=5, \mathrm{~S}=23, \mathrm{~L}=90$ & DFS, and OS & GGO ratio & GGO ratio $\geq 50 \%$ \\
\hline Shi CL (5) & 2011 & 185 & 54 (median) & $127(68.6 \%)$ & Clinical IA $(\leq 2 \mathrm{~cm})$ & 7 & NSCLC & $W+S=36, L=149$ & os & GGO & Pure GGO \\
\hline Okada M (6) & 2011 & 502 & 65 (mean) & $223(44.4 \%)$ & Clinical IA $(\leq 3 \mathrm{~cm})$ & 7 & $A D$ & Unknown (RO resection) & DFS & GGO ratio, TDR, and SUVmax & GGO ratios $\geq 20 \%$, TDR $\geq 30 \%$, and SUVmax $\leq 2.5$ \\
\hline Shimada Y (7) & 2012 & 363 & unknown & $187(51.5 \%)$ & Clinical IA $(\leq 2 \mathrm{~cm})$ & 7 & NSCLC & $S=20, L=343$ & DFS & TDR & $\mathrm{TDR} \geq 50 \%$ without spiculation \\
\hline Koike T (8) & 2013 & 328 & 71 (median) & $196(59.8 \%)$ & Clinical IA $(\leq 3 \mathrm{~cm})$ & 7 & NSCLC & $W=112, S=216$ & css & WTS, and CTR & WTS, and CTR (both: only univariate analysis) \\
\hline Nitadori J (9) & 2013 & 181 & 70 (median) & $61(33.7 \%)$ & Clinical IA $(\leq 2 \mathrm{~cm})$ & 7 & $A D$ & $W=124, S=57$ & Recurrence rate & CTR, and SUVmax & CTR $<0.25$, SUVmax $<2.2$ \\
\hline Tsutani Y (10) & 2013 & 610 & 66 (median) & $268(43.9 \%)$ & Clinical IA $(\leq 3 \mathrm{~cm})$ & 7 & $A D$ & $W=137, S=97, L=376$ & RFS & WTS $(\leq 2$ or $2-3 \mathrm{~cm})$, and CS & cs \\
\hline Kishimoto M (11) & 2014 & 169 & 67 (mean) & $114(67.5 \%)$ & Pathological IA $(\leq 3 \mathrm{~cm})$ & 7 & NSCLC & $S=32, L=137$ & DFS & SUV, and CTR & $\begin{array}{l}\text { SUVmax }<2.5 \text {, and CTR }<0.5 \text { (CTR }<0.5 \text { : only } \\
\text { univariate analysis) }\end{array}$ \\
\hline Sakao Y (12) & 2014 & 176 & 61 (median) & $77(43.8 \%)$ & Clinical IA $(1-3 \mathrm{~cm})$ & 7 & $A D$ & $L=176$ & DFS & WTS, MD, and TDR & MD \\
\hline Sawabata N (13) & 2014 & 110 & 69 (median) & $63(57.3 \%)$ & Clinical I $(\leq 5 \mathrm{~cm})$ & 7 & NSCLC & $S=7, L=103$ & RFS & WTS, CS, and SUVmax & cs \\
\hline Yano M (14) & 2015 & 1737 & 64 (median) & $787(45.3 \%)$ & Clinical IA $(\leq 3 \mathrm{~cm})$ & 7 & NSCLC & $W=643, S=1094$ & DFS & CTR & $\mathrm{CTR} \leq 0.25$ \\
\hline Hattori A (15) & 2015 & 71 & 65 (mean) & $29(40.8 \%)$ & Clinical $I A(<1 \mathrm{~cm})$ & 7 & NSCLC & $W=24, S=20, L=27$ & DFS, and OS & Presence of GGO, SUVmax $\leq 2.5$ & Presence of GGO, and SUVmax $\leq 2.5$ (cT1a) \\
\hline Cho JH (16) & 2015 & 97 & 60 (mean) & $43(44.3 \%)$ & Clinical IA $(\leq 3 \mathrm{~cm})$ & 7 & $A D$ & $W=97$ & RFS, and OS & CTR & $\mathrm{CTR} \leq 0.25$ \\
\hline Takenaka T (17) & 2016 & 255 & 66 (mean) & $129(50.6 \%)$ & Clinical IA $(\leq 3 \mathrm{~cm})$ & 7 & NSCLC & $\mathrm{L}=255$ & DFS, and OS & Presence of GGO, and CS & CS (T category) \\
\hline Yano M (18) & 2016 & 1710 & 63 (mean) & $772(45.1 \%)$ & Clinical IA $(\leq 3 \mathrm{~cm})$ & 7 & NSCLC & $W=637, L=1,073$ & DFS & CTR & AD with $\mathrm{CTR} \leq 0.25$ \\
\hline Hattori A (19) & 2017 & 328 & unknown & $122(37.2 \%)$ & Clinical IA $(\leq 1 \mathrm{~cm})$ & 8 & NSCLC & $W=105, S=110, L=113$ & DFS, and OS & Presence of GGO & Presence of GGO \\
\hline Hattori A (20) & 2017 & 497 & unknown & $267(53.7 \%)$ & Clinical IA $(\leq 3 \mathrm{~cm})$ & 7 & NSCLC & $\mathrm{W}=33, \mathrm{~S}=75, \mathrm{~L}=389$ & os & $\begin{array}{l}\text { Presence of GGO, WTS ( } \leq 20 \text { or } \\
21-30 \mathrm{~mm}) \text {, and CS }(\leq 20 \text { or } 21-30 \mathrm{~mm})\end{array}$ & Presence of GGO \\
\hline Ye T (21) & 2018 & 736 & unknown & $210(28.5 \%)$ & Clinical IA $(\leq 3 \mathrm{~cm})$ & 8 & $A D$ & $\mathrm{~W}=474, \mathrm{~S}=89, \mathrm{~L}=278$ (841 nodules) & DFS, and OS & WTS, CTR, and CS & CTR, and CS (both: only univariate analysis) \\
\hline Su H (22) & 2018 & 247 & unknown & $113(45.7 \%)$ & Clinical IA $(\leq 3 \mathrm{~cm})$ & 7 & $A D$ & $W=153, S=94$ & RFS & WTS, and CS (T category) & CS (T category) \\
\hline Su H (23) & 2018 & 245 & unknown & $89(36.3 \%)$ & Clinical IA $(\leq 3 \mathrm{~cm})$ & 8 & $A D$ & $W+S=55, L=190$ & RFS & CTR & $\mathrm{CTR} \leq 0.5$ \\
\hline Goto M (24) & 2019 & 99 & 68 (median) & $50(50.5 \%)$ & Clinical $\mathrm{IA}(\leq 1 \mathrm{~cm})$ & 8 & NSCLC & $\mathrm{L}=a b o u t$ half & RFS, and OS & $\mathrm{CS}$ (T category) & $\mathrm{CS}$ (T category) \\
\hline Fu F (25) & 2019 & 2010 & unknown & $1023(50.9 \%)$ & Pathological IA $(\leq 3 \mathrm{~cm})$ & 8 & NSCLC & $W=79, S=73, L$ or greater $=1858$ & RFS & Presence of GGO & Presence of GGO \\
\hline Katsumata S (26) & 2019 & 744 & unknown & $411(55.2 \%)$ & Clinical IA $(\leq 3 \mathrm{~cm})$ & 8 & NSCLC & L or greater $=744$ & os & CS ( $T$ category), and CTR & CS (cT1a or less), and CTR $\leq 0.5$ \\
\hline Kim H (1) & 2019 & 691 & 63 (median) & $281(40.7 \%)$ & Clinical IA $(\leq 3 \mathrm{~cm})$ & 8 & $A D$ & $\mathrm{~L}=691$ & DFS & $\mathrm{CS}, \mathrm{CTR}$, and TDR & $\mathrm{CS}$ (T category) \\
\hline Kim H (27) & 2019 & 506 & 62 (median) & $200(39.5 \%)$ & Clinical IA $(\leq 3 \mathrm{~cm})$ & 8 & $A D$ & $L=506$ & DFS & WTS & WTS \\
\hline Ye T (28) & 2019 & 911 & 56 (mean) & $277(30.4 \%)$ & Clinical IA $(\leq 3 \mathrm{~cm})$ & 8 & $A D$ & $\mathrm{~W}=456, \mathrm{~S}=97, \mathrm{~L}=435$ (988 nodules) & RFS, and OS & WTS, CTR, and CS & None of them \\
\hline Miyoshi T (29) & 2019 & 809 & 67 (median) & $405(50 \%)$ & Pathological IA $(\leq 3 \mathrm{~cm})$ & 8 & $A D$ & $L=809$ & os & Presence of GGO & Presence of GGO \\
\hline Hattori A (30) & 2019 & 634 & unknown & $291(45.9 \%)$ & Clinical IA $(\leq 3 \mathrm{~cm})$ & 8 & AD & $\mathrm{W}+\mathrm{S}=279, \mathrm{~L}=355$ & RFS, and OS & Presence of GGO, CS & Presence of GGO, and CS \\
\hline Chiang XH (31) & 2020 & 1035 & 59.5 (mean) & $351(33.9 \%)$ & Clinical IA $(\leq 3 \mathrm{~cm})$ & 8 & AD & $W=470, S=134, L=431$ & DFS & WTS, CS, and CTR & cs \\
\hline Kim H (32) & 2020 & 744 & 63 (median) & $324(43.5 \%)$ & Clinical IA $(\leq 3 \mathrm{~cm})$ & 8 & $A D$ & Unknown (RO resection) & os & Presence of GGO, and CS & $\mathrm{CS}$ (T category) \\
\hline Akın Kabalak (33) & 2020 & 156 & 62 (mean) & $121(77.6 \%)$ & Pathological IA $(\leq 3 \mathrm{~cm})$ & 7 & $A D$ & $W+S=19, L$ or greater $=137$ & PFS, and OS & WTS (T1 vs. T2a), CTR, and SUVmax & SUVmax (PFS), and CTR <0.5 (OS) \\
\hline Kuroda $\mathrm{H}(34)$ & 2020 & 260 & 64 (median) & $128(49.2 \%)$ & Clinical IA $(\leq 3 \mathrm{~cm})$ & 8 & NSCLC & $S=14, L=246$ & DFS, and OS & $\mathrm{CS}$ ( $\mathrm{T}$ category), CTR, TDR, and MD & $\mathrm{CS}$ ( $\mathrm{T}$ category), and $\mathrm{MD}$ \\
\hline
\end{tabular}


evaluated the effect of the presence of GGO and CS (T category) on DFS and OS. Part solid tumors had better OS and DFS rates than solid tumors (OS: $\mathrm{P}=0.019$, DFS: $\mathrm{P}=0.011$ ) and part-solid tumors (OS 83.2\%; DFS 78.2\%) groups. After matching the cases according to the size of the solid component, the presence of the GGO was not a prognostic factor (OS, $\mathrm{P}=0.39$; $\mathrm{DFS}, \mathrm{P}=0.37$ ). However, CS (T category) was an independent prognostic factor (OS, $\mathrm{P}<0.001$; DFS, $\mathrm{P}<0.001)$.

In 2020, we examined the effect of radiological parameters (CS, CTR, TDR, and MD) on DFS and OS in 260 patients $(\leq 3 \mathrm{~cm})$ with c-stage IA NSCLC under TNM 8 , who underwent thoracotomy between 2006 and 2010 (34). The estimated HR for DFS and OS were calculated according to the clinical T category, CTR, TDR, and MD. In this study, CS (T category) and MD were superior prognostic factors for NSCLC compared with CTR or TDR. In addition, we examined the effect of MD on DFS and OS in 245 patients $(\leq 4 \mathrm{~cm})$ with c-stage I NSCLC under TNM 8 [including minimally invasive adenocarcinoma (MIA) in 22 patients $(9.0 \%)$ ] who underwent thoracoscopic surgery between 2013 and 2016 (35). A significant difference in DFS between $\mathrm{MD} \leq 5 \mathrm{~mm}$ and $\mathrm{MD}>20 \mathrm{~mm}$ was observed $(\mathrm{P}=0.04)$; however, there was no difference in OS.

\section{NSCLC in pathological stage T1NOMO}

Two studies focused on clinical NSCLCs. The TNM version 7 was used in one study, and version 8 in the other report. The maximum standardized uptake value (SUVmax) on PET/CT and the presence of GGO were potential prognostic factors.

Kishimoto analyzed 169 patients with lung cancer $\leq 3 \mathrm{~cm}$ in TNM version 7 (11). Twenty-eight patients $(16.6 \%)$ had a recurrence of NSCLCs. The DFS was significantly reduced for patients with $S U V \max$ of $\geq 2.5$, compared with those with SUVmax of $<2.5(\mathrm{P}<0.001)$ or when the CTR was $\geq 50 \%$ compared with when the CTR was $<50 \%(\mathrm{P}=0.03)$. A Cox hazards ratio model showed that SUVmax was an independent predictor of recurrence (HR =1.324; $\mathrm{P}<0.001$ ). Thus, SUVmax on PET/CT was a significant biomarker for lung cancer prognosis and was superior to the CTR in predicting postoperative recurrence.

Fu et al. analyzed 2010 patients with NSCLC pathological stage IA $(\leq 3 \mathrm{~cm})$ under TNM 8 (25). Cox multivariate analyses revealed that the absence of GGO was a strong independent risk factor for worse relapsefree survival (RFS) (HR: 0.426, $\mathrm{P}<0.001$ ). Moreover, the
5 -year OS differed significantly between the solid and partsolid tumors $(83.4 \%$ vs. $94.9 \%, \mathrm{P}<0.001)$ but not between the part-solid and pure-GGO tumors $(94.9 \%$ vs. $98.5 \%$, $\mathrm{P}=0.115)$.

\section{Adenocarcinoma}

Sixteen publications focused on adenocarcinoma. The TNM 7 was used in seven studies, and version 8 in 9 studies. In recent years, studies on adenocarcinoma have increased compared to those on NSCLC. The median number of patients was 558 (range, $97-1,035$ patients), the median proportion of males was $43.7 \%$ (range, $28.5 \%$ $77.6 \%)$. The median or mean age range was $56-70$ years. The radiological parameters evaluated in these studies were 8 WTS, 8 CS including T category, 8 CTR, 3 TDR, 1 MD, 3 presence of GGO, and 1 GGO ratio. After excluding pure GGO in univariate analysis, the final prognostic parameters were 1 WTS, 6 CS including T category, 5 CTR (1 result from a study of NSCLC), 1 TDR, $1 \mathrm{MD}, 2$ presence of GGO, and 1 GGO ratio. For adenocarcinoma, CS, including T category and CTR are potential factors for better prognosis.

\section{MIA}

Patients with MIA have nearly $100 \%$ disease-specific survival if the tumor is completely resected. MIA has no lymphatic, vascular, or pleural invasion or necrosis (36).

Suzuki reported that lung adenocarcinoma $(\leq 2.0 \mathrm{~cm})$ with consolidation $(<0.25 \mathrm{~cm})$ corresponds to the cT1 mi category per the $8^{\text {th }}$ TNM classification (37). Hayashi retrospectively evaluated 188 patients with surgically resected T1 lung adenocarcinoma (38). Solid component size ( $\mathrm{MD}>5 \mathrm{~mm}$ or $\mathrm{CD}>8 \mathrm{~mm}$ ) could predict lymph node metastasis and local invasiveness. However, it was difficult to determine the solid component using HRCT because of the normal lung tissues in the background. We previously suggested that the prognostic effect of MD is superior to that of WTS or CD for lung adenocarcinoma.

In 2016, Sakakura evaluated 360 completely resected adenocarcinomas from 2012 to 2015 that were staged as cT1a-1b-2aN0 based on TNM 7; however, two debatable issues were identified (39). First, the pathological invasive size correlated well with both $\mathrm{CD}\left(\mathrm{r}^{2}=0.710\right)$ and $\mathrm{MD}$ $\left(\mathrm{r}^{2}=0.743\right)$, and moderately with WTS $\left(\mathrm{r}^{2}=0.514\right)$. MD $\leq 2 \mathrm{~mm}$ predicted MIA with high specificity (94.5\%). 
Second, the pathological invasive size included both the benign scar and the malignant component; however, no differentiation between CD and MD could be made.

\section{Adenocarcinoma in clinical stage T1NOMO}

Fourteen publications focused on clinical stage I adenocarcinoma. TNM 7 was used in 6 studies, and version 8 in 8 studies. After excluding available parameters in the univariate analysis, the factors for better prognosis were 1 WTS, 6 CS including T category, 4 CTR, 1 TDR, 1 MD, 1 presence of GGO, and 1 GGO ratio. CS, including $\mathrm{T}$ category, was a potential factor for better prognosis.

\section{Total adenocarcinomas}

In the cT1N0M0 stage, according to TNM 7, CTR was significantly associated with OS. Asamura reported that the 5 -year OS was $96.7 \%$ for adenocarcinomas with CTR $\leq 50 \%$ and $88.9 \%$ for adenocarcinomas with CTR $>50 \%(\mathrm{P}<0.001)(40)$. Nitadori reviewed 181 patients who underwent limited resection for lung adenocarcinoma $\leq 2 \mathrm{~cm}$ (9). Patients with low CTR ( $\mathrm{n}=15)$ had a significantly lower 5 -year recurrence rate than patients with a high CTR $(\mathrm{n}=166)$ [5-year cumulative incidence of recurrence (CIR), 0 vs. $33 \% ; \mathrm{P}=0.015$ ]. A similar outcome was observed in patients with a low SUVmax $(\mathrm{n}=86)$ compared to those with a high SUVmax (n=95; 5-year CIR, 18\% vs. 40\%; $\mathrm{P}=0.002$ ). Furthermore, in the high CTR group, the risk of recurrence was further stratified by SUVmax [5-year CIR, $22 \%$ (low) vs. 40\% (high); $\mathrm{P}=0.018$ ]. This shows that CTR and SUVmax might be indicators of tumor aggressiveness.

In 2020, Kim studied 744 patients with clinical stage IA adenocarcinoma $(\leq 3 \mathrm{~cm})$ under TNM 8 (32). T categories were significantly associated with overall survival (HR of cT1b: 2.33, $\mathrm{P}=0.033$; HR of cT1c: $5.74, \mathrm{P}<0.001)$. The T-categorization system is valid for PSNs and solid nodules. The multivariable mixture cure model revealed that solid nodules were associated with a decreased probability of long-term survival ( $\mathrm{OR}=0.40, \mathrm{P}=0.030$ ). $\mathrm{PSN}$ s are prognostic factors associated with long-term survival. Survival curves were adjusted for age, sex, nodule location, surgical mode, history of malignancy, family history of lung cancer, smoking status, and clinical T category. After adjustment, the difference was insignificant (OS, $\mathrm{P}=0.189$ ). Moreover, when solid portion size was used as one of the inputs in the Cox regression model instead of the $\mathrm{cT}$ category, the HR of nodule type was not significant (HR: 1.26, $\mathrm{P}=0.344)$. The solid portion size was a significant predictor of OS (HR: 1.10, $\mathrm{P}<0.001)$. Chiang reviewed 1,035 patients with clinical stage IA adenocarcinoma $(\leq 3 \mathrm{~cm})$ under TNM 8 (31). They evaluated the effect of WTS, CS, and CTR on DFS. Multivariate analysis revealed that CS and serum carcinoembryonic antigen levels were independent risk factors for tumor recurrence. Su analyzed 247 patients with clinical IA-stage lung adenocarcinoma under TNM 7 (22). They compared WTS (T category according to TNM version 7) and CS (T category according to TNM version 8) for RFS. They concluded that CS was statistically better than WTS in predicting the recurrence of lung adenocarcinoma.

Hattori evaluated the prognostic effect of GGO at each clinical stage (30). The cohort included 546 patients with c-stage IA, excluding T1mi lesions under TNM 8. All tumors were subclassified into the GGO group $(0<\mathrm{CTR}$ $<1.0)$ or solid group $(\mathrm{CTR}=1.0)$ at each stage. The 5 -year OS was significantly different between the GGO and solid tumor groups (IA1: $97.8 \%$ vs. $86.6 \%, \mathrm{P}<0.026$; IA2: $89.3 \%$ vs. $75.2 \%, \mathrm{P}<0.007$; IA3: $88.5 \%$ vs. $62.3 \%, \mathrm{P}<0.003)$. They concluded that the presence of GGO was an independent and significant prognostic factor $(\mathrm{P}<0.024)$. Using the Cox proportional hazard model, CS (HR: 1.040, $\mathrm{P}=0.038$ ) and presence of GGO (HR: $0.485, \mathrm{P}=0.024$ ) were predictive factors for survival in the multivariate analyses.

In 2014, Sakao evaluated 176 patients with clinical stage IA (1-3 cm) under TNM 7 (12), and reported that MD $\leq 5 \mathrm{~mm}$ had no vascular, lymphatic, or pleural invasion, which was almost equivalent to MIA. The areas under the curve for recurrence of the tumor were $0.76,0.73$, and 0.65 , for MD, TDR, and CD, respectively. The 5-year DFS rates according to the $\mathrm{MD}$ were $98.1 \%$ for tumors $<10 \mathrm{~mm}$ $(\mathrm{n}=52), 71.0 \%$ for tumors $11-15 \mathrm{~mm}(\mathrm{n}=52)$, and $49.0 \%$ for tumors $>15 \mathrm{~mm}(\mathrm{n}=72)(\mathrm{P}<0.01)$.

\section{Part solid nodules}

Su analyzed 254 patients with clinical IA stage lung adenocarcinoma with ground-glass nodules (GGNs) under TNM 8 (23). Of these patients, 179 (73\%) had GGOpredominant nodules (CTR $\leq 0.5)$, and the remaining 66 $(27 \%)$ had solid-predominant nodules (CTR $>0.5$ ). Patients with solid-predominant nodules had a higher incidence of larger tumor size $(2-3 \mathrm{~cm} ; 35 \%$ vs. $12 \% ; \mathrm{P}<0.001)$. Tumor size $(2-3 \mathrm{~cm} v s . \leq 2 \mathrm{~cm})$ was not an independent risk factor for recurrence of the tumor in univariate analysis $(\mathrm{P}=0.288)$. Multivariate logistic regression analysis revealed that CTR $(>0.5, \leq 0.5)$ was an independent risk factor for recurrence of 
the tumor (HR: 9.47, $\mathrm{P}=0.009$ ). Among patients with a $0.5<$ CTR $<1$, the 5 -year RFS was significantly higher in the lobectomy versus sublober resection group $(92 \%$ vs. $60 \%$, $\mathrm{P}=0.002$ ).

Ye studied 736 patients and 841 GGNs $\leq 3 \mathrm{~cm}$ in TNM 8 (21). They evaluated the prognostic effect of CTR, CS, and WTS. CTR and CS had a prognostic effect in univariate analysis but not in multivariate analysis. The results were as follows: CTR (multivariable), HR 2.79, $\mathrm{P}=0.565$; WTS $(20<$ size $<30 \mathrm{~mm}$, univariable), HR 2.15, $\mathrm{P}=0.094$; CS $(10<$ size $<30 \mathrm{~mm}$, multivariable), HR 1.22, $\mathrm{P}=0.754$. The authors noted that none of the factors could predict the prognosis of the tumor. Ye evaluated the prognosis of clinical stage IA $(\leq 3 \mathrm{~cm})$ adenocarcinoma according to TNM 8, including 329 PSNs, 501 pure GGNs, and 158 pure solid nodules (28). In this study, PSN had better 5-year DFS and lung cancer-specific (LCS) OS than pure solid tumors with similar clinical $\mathrm{T}$ stages (cT1b and cT1c). WTS could predict the 5-year DFS and OS of patients with pure solid tumors (WTS = CS). In the PSN group, CTR, CS, and WTS could predict pathologically invasive adenocarcinoma, but not its prognosis. They concluded that part-solid lung adenocarcinoma should be considered a special clinical subtype, similar to a previous report.

Hattori recruited a cohort of 546 patients with c-stage IA, excluding T1mi lesions under TNM version 8 , and evaluated the prognosis of GGOs at each clinical stage (30). In the subgroup, they compared the survival outcomes of predominant GGO (CTR $\leq 0.5)$ versus non-predominant GGO tumors $(0.5<$ CTR $<1.0)$. Overall, no significant difference was observed between the predominant and nonpredominant GGO tumors ( $98.2 \%$ vs. $91.5 \%, \mathrm{P}=0.064)$. Moreover, no significant differences were observed between predominant GGO and non-predominant GGO in the cT1a and cT1b GGO tumors (cT1a: $97.9 \%$ vs. $100 \%$, $\mathrm{P}=0.983$; and cT1b: $100 \%$ vs. $88.8 \%, \mathrm{P}=0.373)$. In contrast, the survival outcomes between non-predominant GGO and solid tumors $(\mathrm{CTR}=1.0)$ were significantly different in both cT1a and cT1b lesions despite the radiological solid predominance (cT1a: $100 \%$ vs. $86.6 \%, \mathrm{P}=0.046$ and cT1b: $88.8 \%$ vs. $75.2 \%, \mathrm{P}=0.011$ ).

Kim studied 352 PSNs with clinical stage T1N0M0 using TNM 8 (1). All Cox models revealed that patient age and clinical $\mathrm{T}$ category were independent prognostic factors for DFS in PSNs. In the other subgroup (PSNs from cT1mi/cT1a to cT1b), CTR and TDR were not significantly associated with DFS after adjusting for other prognostic factors (all $\mathrm{P}>0.05$ ).

Additionally, Kim et al. reported another retrospective study that evaluated 506 patients with stage IA lung adenocarcinoma appearing as PSNs (27). Multivariate Cox regression analysis demonstrated that the WTS of cT1b (interaction term; HR $=1.091 ; 95 \%$ CI: 1.015, 1.173; $\mathrm{P}=0.019)$ and WTS of cT1c (HR $=68.436$; 95\% CI: 2.797, $1,674.415 ; \mathrm{P}=0.010$ ) were independent risk factors for tumor recurrence. When patients with $\mathrm{cT} 1 \mathrm{~b}$ were stratified based on a WTS size cutoff of $3.0 \mathrm{~cm}$, PSNs with WTS $>3.0 \mathrm{~cm}$ had a significantly worse outcome (HR $=3.796 ; 95 \% \mathrm{CI}$ : 1.006, 14.317; $\mathrm{P}=0.049)$. No significant difference was observed in the risk of recurrence between cT1b with WTS $>3.0 \mathrm{~cm}$ and $\mathrm{cT} 1 \mathrm{c}(\mathrm{P}=0.915)$. In cT1a and cT1c, WTS was not significantly associated with recurrence $(\mathrm{P}=0.680$ and 0.224 , respectively). These outcomes in c $\mathrm{T} 1$ may be divided into any T1a and T1b WTS $<3 \mathrm{~cm}$ group $v$ s. T1b WTS $\geq 3 \mathrm{~cm}$ and any $\mathrm{T} 1 \mathrm{c}$ group.

\section{Adenocarcinoma in pathological stage T1NOMO}

Imaging findings may be useful for pathological diagnosis and pre-diagnosis. Two studies focused on pathological adenocarcinoma; one study used TNM 7, while the other used TNM 8. Finally, the available indicators of better prognosis were 1 CTR and 1 presence of GGO.

Akın evaluated 156 patients with pathologic stage IA adenocarcinoma $\leq 3 \mathrm{~cm}$ under TNM 7 (33). They analyzed the prognostic effect of WTS (T1 vs. T2a), CTR, and SUVmax for PFS and OS. CTR $\geq 0.5$ was a predictive factor for OS and PFS (OS: $\mathrm{P}=0.002$ and PFS: $\mathrm{P}=0.005$ ). Multivariate analysis using a Cox proportional hazards model showed that resection type and CTR were better predictors of OS (resection type: HR: $2.21, \mathrm{P}=0.045$ and CTR: HR: $0.44, \mathrm{P}=0.045)$. SUVmax was not a predictor of OS (HR: 0.99, P=0.96). For PFS, surgery type and SUVmax were significant predictors in multivariate analyses (surgery type, HR: 3.56, $\mathrm{P}=0.001$ and SUVmax, HR: 1.31, $\mathrm{P}=0.002$ ).

Miyoshi evaluated 809 patients with pathologic stage IA adenocarcinoma $\leq 3 \mathrm{~cm}$ under TNM 8 , including 465 (57\%) with PSNs and 344 (43\%) with solid nodules (29). On final pathology, lepidic adenocarcinoma was identified in 445 (96\%) cases with PSNs and 239 (69\%) cases with solid nodules. The survival rate of patients with GGNs was significantly higher than that of patients without GGO components (5-year OS, $97 \%$ vs. $84 \%, \mathrm{P}<0.0001)$. In solid nodules, there was no significant prognostic difference between patients with and without a lepidic component 
(5-year OS, $87 \%$ vs. $79 \%, \mathrm{P}=0.09$ ), and a similar tendency was observed in $\mathrm{pT} 1 \mathrm{~b}$ and $\mathrm{pT} 1 \mathrm{c}$ lesions when the pathologic $\mathrm{T}$ status was identical. On multivariable analysis of all nodules, solid appearance on chest $\mathrm{CT}$ was an independent prognostic factor ( $\mathrm{HR}=1.74, \mathrm{P}=0.019$ ), although the pathologic invasive size and pathologic lepidic growth component were not.

\section{Solid tumor in clinical T1NOMO}

Solid adenocarcinoma, squamous cell carcinoma (SCC), adenosquamous cell carcinoma (ASC), and neuroendocrine tumors (NETs) usually appear as solid nodules on CT.

Fu et al. analyzed 2,010 patients with NSCLC $\leq 3 \mathrm{~cm}$ under TNM 8 (25). The absence of GGO was a strong independent risk factor for worse RFS $(\mathrm{P}<0.001)$. For the solid group, the adenocarcinoma subtype was not a prognostic factor for RFS $(\mathrm{P}=0.162)$. Hattori evaluated the prognostic effect of GGO among 546 patients with c-stage IA NSCLC, excluding T1mi lesions under TNM 8 (30). The 5 -year OS was significantly different between the GGO $(0<$ CTR $<1.0)$ and solid tumor $($ CTR $=1.0)$ groups. Additionally, Hattori evaluated 71 patients with NSCLC $(\leq 1 \mathrm{~cm}$ NSCLC) using TNM 7 and 328 patients $(\leq 3 \mathrm{~cm}$ NSCLC) using TNM 8 to determine the effect of GGO on DFS and OS $(15,19)$. These reports show that solid tumors are indicators of poor prognosis.

Ito evaluated 150 patients with solid type NSCLC $<3 \mathrm{~cm}$ in diameter on HRCT (adenocarcinoma, n=106; SCC, $\mathrm{n}=36$; ASC, $\mathrm{n}=8$, who had undergone surgical resection (41). The SUVmax of lung SCC was reported to be higher than that of adenocarcinoma, although a few studies have suggested that it is not a prognostic factor $(42,43)$. However, these previous studies included both subsolid and partly solid tumors. SUVmax was significantly correlated with tumor recurrence $(\mathrm{P}=0.004)$, although tumor size and histopathological type were not correlated with tumor recurrence $(\mathrm{P}=0.502$ and $\mathrm{P}=0.351$, respectively). Finally, sorting these three pathological types of NSCLC into the same classification and selecting from PSN does not cause a problem in prognosis prediction following HRCT.

NETs of the lung constitute approximately $20 \%$ of all primary lung tumors, including typical carcinoid, atypical carcinoid, large cell neuroendocrine carcinoma, and SCC. NETs appear as solitary tumors on CT, and their oncological behaviors are different. $\mathrm{SUV}_{\max }$ on PET-CT was useful in differentiating NETs (44). Jackson evaluated 12,415 cases of NETs from the National Cancer Database (45).
The studies suggested that $\mathrm{T}$ stage and category and histologic grade were important prognostic factors.

\section{Discussion}

CS and MD were equally evaluated in TNM version 7 or 8. In particular, many studies showed that CS was the most useful in predicting malignant behavior regarding NSCLC and PSNs. Ye detected that WTS (= CS) could predict the 5 -year DFS and OS in pure solid adenocarcinoma (28). Staging of stage IA NSCLC with TNM version 8 was highly valid.

Some CT parameters such as CTR, TDR, and GGO ratio were different in the control group between TNM versions 7 and 8 because the T category of version 7 is based on WTS, but version 8 is based on CS. In TNM version 7, tumors with small WTS and large CTR and tumors with large WTS and small CTR could have been assigned to a different control group despite the same invasive size. In TNM-7, CTR, TDR, and GGO ratio encompassed the morphology only, but not the invasive size element. Furthermore, the cutoff values for these parameters were not determined. Some studies using TNM- 8 suggested that CTR $\leq 0.5$ was a better prognostic factor for both NSCLC and adenocarcinoma $(23,26)$.

We examined the effect of radiological parameters (CS, CTR, TDR, and MD) on DFS and OS in patients with c-stage IA NSCLC using TNM-8. The prognostic effect of CS (T category) and MD was superior to that of CTR or TDR (34). These studies included solid tumors. However, recent studies suggested that CTR or TDR for PSNs was not a well-accepted prognostic factor for part solid tumors $(1,8,11,12,21,28,31,34)$. In the PSN group, CTR, CS, and WTS could predict pathologically invasive adenocarcinoma, but not prognosis. Part solid lung adenocarcinoma is considered a special clinical subtype. However, CS, including the $\mathrm{T}$ category, could predict the prognosis of the tumor.

The presence of GGO was evaluated in both part solid tumors and pure solid tumors. Some reports revealed that the malignant behavior of part solid tumors was an indicator of better prognosis for both NSCLC and adenocarcinoma. Takenaka evaluated the effect of the presence of GGO and CS (T category) in NSCLC on DFS and OS (17). After matching according to the size of the solid component, the presence of the GGO was not a prognostic factor (OS, $\mathrm{P}=0.39$; DFS, $\mathrm{P}=0.37$ ). However, CS (T category) was an independent prognostic factor for NSCLC (OS, $\mathrm{P}<0.001$; DFS, $\mathrm{P}<0.001)$. In NSCLC, the prognostic effect of the 
presence of GGOs remains controversial. Kim evaluated the WTS of 506 patients with stage IA lung adenocarcinoma appearing as PSNs (27). They indicated that $\mathrm{c}$ T1 might be divided into any T1a and T1b WTS $<3 \mathrm{~cm}$ group or T1b WTS $\geq 3 \mathrm{~cm}$ and any T1c group. As for WTS in PSNs, further investigations are required to evaluate the effect of large WTS on the prognosis of PSNs.

Our findings indicate that CS and MD are useful parameters for predicting invasive size and prognosis of NSCLC (34). CS and MD are superior to pathological invasive size in cases with pathological lymph node involvement but not in cases with lymphatic/vascular/ pleural invasion (46). Notably, CS and MD can predict the outcome of segmentectomy for cT1mi. However, we could not differentiate between benign scars and malignant components on HRCT. Therefore, multimodal imaging, including positron emission tomography-CT, is needed to comprehensively predict the malignancy of the tumor preoperatively.

In this study, we evaluated the current literature on the prognostic radiological tools using CT. Predicting oncological behaviors is important for surgical planning and aggressive surveillance of the tumor. Finally, we considered a high risk of tumor recurrence indicating a possible need for adjuvant therapy. In clinical and pathological T1N0Staged NSCLC, pure solid tumor and larger CS (T category) were associated with DFS $(17,20,25)$. In lung adenocarcinoma, CS was also predicting the recurrence of lung adenocarcinoma $(22,27,31)$. However, several authors suggested that part-solid tumors should be considered a special clinical subtype with better prognosis for clinical and pathological T1N0-Staged lung adenocarcinoma $(21,28-30)$. In summary, larger pure solid tumors were possible to indicate aggressive adjuvant or neoadjuvant therapy in this setting.

\section{Limitations}

This review has several limitations. First, there is high heterogeneity due to variations in patient background and the number of cases in each study. Second, errors may occur depending on the researcher measuring the tumor, for example, distinguishing between CS and GGO components. Third, the outcomes of pathological lymph node metastasis were not evaluated in this review. Although, T1 lung cancers could be treated with surgery or SBRT radiation therapy, we evaluated prognostic radiological parameters only for surgery according to our exclusion criteria.

\section{Conclusions}

This review shows that CS, MD, and the presence of GGO are optimal prognostic radiological tools for cT1N0staged NSCLC. However, CTR or TDR for PSNs is not a well-accepted prognostic factor. Further investigations are required to differentiate between benign scars and malignant components on HRCT and evaluate the prognosis of PSNs $(1<\mathrm{CS} \leq 2 \mathrm{~cm})$ with large WTS.

\section{Acknowledgments}

The authors would like to thank Editage (https://www. editage.jp) for English language review.

\section{Footnote}

Reporting Checklist: The authors have completed the Narrative Review reporting checklist. Available at http:// dx.doi.org/10.21037/jtd-20-3380

Conflicts of Interest: Both authors have completed the ICMJE uniform disclosure form (available at http://dx.doi. org/10.21037/jtd-20-3380). The authors have no conflicts of interest to declare.

Ethical Statement: The authors are accountable for all aspects of the work in ensuring that questions related to the accuracy or integrity of any part of the work are appropriately investigated and resolved.

Open Access Statement: This is an Open Access article distributed in accordance with the Creative Commons Attribution-NonCommercial-NoDerivs 4.0 International License (CC BY-NC-ND 4.0), which permits the noncommercial replication and distribution of the article with the strict proviso that no changes or edits are made and the original work is properly cited (including links to both the formal publication through the relevant DOI and the license). See: https://creativecommons.org/licenses/by-nc-nd/4.0/.

\section{References}

1. Kim H, Goo JM, Kim YT, et al. Consolidation-to-tumor ratio and tumor disappearance ratio are not independent prognostic factors for the patients with resected lung adenocarcinomas. Lung Cancer 2019;137:123-8.

2. Nakamura K, Saji H, Nakajima R, et al. A phase 
III randomized trial of lobectomy versus limited resection for small-sized peripheral non-small cell lung cancer (JCOG0802/WJOG4607L). Jpn J Clin Oncol 2010;40:271-4.

3. Ito H, Suzuki K, Mizutani T, et al. Long-term survival outcome after lobectomy in patients with clinical T1 N0 lung cancer. J Thorac Cardiovasc Surg 2020. [Epub ahead of print]. doi: 10.1016/j.jtcvs.2019.12.072.

4. Inoue $\mathrm{M}$, Minami $\mathrm{M}$, Sawabata $\mathrm{N}$, et al. Clinical outcome of resected solid-type small-sized c-stage IA non-small cell lung cancer. Eur J Cardiothorac Surg 2010;37:1445-9.

5. Shi CL, Zhang XY, Han BH, et al. A clinicopathological study of resected non-small cell lung cancers $2 \mathrm{~cm}$ or less in diameter: a prognostic assessment. Med Oncol 2011;28:1441-6.

6. Okada M, Nakayama H, Okumura S, et al. Multicenter analysis of high-resolution computed tomography and positron emission tomography/computed tomography findings to choose therapeutic strategies for clinical stage IA lung adenocarcinoma. J Thorac Cardiovasc Surg 2011;141:1384-91.

7. Shimada Y, Yoshida J, Hishida T, et al. Predictive factors of pathologically proven noninvasive tumor characteristics in T1aN0M0 peripheral non-small cell lung cancer. Chest 2012;141:1003-9.

8. Koike T, Koike T, Yoshiya K, et al. Risk factor analysis of locoregional recurrence after sublobar resection in patients with clinical stage IA non-small cell lung cancer. J Thorac Cardiovasc Surg 2013;146:372-8.

9. Nitadori J, Bograd AJ, Morales EA, et al. Preoperative consolidation-to-tumor ratio and SUVmax stratify the risk of recurrence in patients undergoing limited resection for lung adenocarcinoma $\leq 2 \mathrm{~cm}$. Ann Surg Oncol 2013;20:4282-8.

10. Tsutani $Y$, Miyata $Y$, Nakayama $H$, et al. Solid tumor size on high-resolution computed tomography and maximum standardized uptake on positron emission tomography for new clinical $\mathrm{T}$ descriptors with $\mathrm{T} 1$ lung adenocarcinoma. Ann Oncol 2013;24:2376-81.

11. Kishimoto M, Iwano S, Ito S, et al. Prognostic evaluations of small size lung cancers by 18F-FDG PET/CT and thinsection CT. Lung Cancer 2014;86:180-4.

12. Sakao Y, Kuroda H, Mun M, et al. Prognostic significance of tumor size of small lung adenocarcinomas evaluated with mediastinal window settings on computed tomography. PLoS One 2014;9:e110305.

13. Sawabata N, Kanzaki R, Sakamoto T, et al. Clinical predictor of pre- or minimally invasive pulmonary adenocarcinoma: possibility of sub-classification of clinical T1a. Eur J Cardiothorac Surg 2014;45:256-61.

14. Yano M, Yoshida J, Koike T, et al. Survival of 1737 lobectomy-tolerable patients who underwent limited resection for cStage IA non-small-cell lung cancer. Eur J Cardiothorac Surg 2015;47:135-42.

15. Hattori A, Suzuki K, Matsunaga T, et al. What is the appropriate operative strategy for radiologically solid tumours in subcentimetre lung cancer patients? Eur J Cardiothorac Surg 2015;47:244-9.

16. Cho JH, Choi YS, Kim J, et al. Long-term outcomes of wedge resection for pulmonary ground-glass opacity nodules. Ann Thorac Surg 2015;99:218-22.

17. Takenaka T, Yamazaki K, Miura N, et al. The Prognostic Impact of Tumor Volume in Patients with Clinical Stage IA Non-Small Cell Lung Cancer. J Thorac Oncol 2016;11:1074-80.

18. Yano M, Yoshida J, Koike T, et al. The Outcomes of a Limited Resection for Non-Small Cell Lung Cancer Based on Differences in Pathology. World J Surg 2016;40:2688-97.

19. Hattori A, Matsunaga T, Hayashi T, et al. Prognostic Impact of the Findings on Thin-Section Computed Tomography in Patients with Subcentimeter Non-Small Cell Lung Cancer. J Thorac Oncol 2017;12:954-62.

20. Hattori A, Matsunaga T, Takamochi K, et al. Importance of Ground Glass Opacity Component in Clinical Stage IA Radiologic Invasive Lung Cancer. Ann Thorac Surg 2017;104:313-20.

21. Ye T, Deng L, Xiang J, et al. Predictors of Pathologic Tumor Invasion and Prognosis for Ground Glass Opacity Featured Lung Adenocarcinoma. Ann Thorac Surg 2018;106:1682-90.

22. Su H, Dai C, She Y, et al. Which T descriptor is more predictive of recurrence after sublobar resection: whole tumour size versus solid component size? Eur J Cardiothorac Surg 2018;54:1028-36.

23. Su H, Dai C, Xie H, et al. Risk factors of recurrence in patients with clinical stage IA adenocarcinoma presented as ground-glass nodule. Clin Lung Cancer 2018;19:e609-17.

24. Goto M, Kawaguchi K, Fukui T, et al. Verification of T descriptor with consolidation size for sub-centimeter nonsmall cell lung cancer. Surg Today 2019;49:907-12.

25. Fu F, Zhang Y, Wen Z, et al. Distinct Prognostic Factors in Patients with Stage I Non-Small Cell Lung Cancer with Radiologic Part-Solid or Solid Lesions. J Thorac Oncol 2019;14:2133-42.

26. Katsumata S, Aokage K, Nakasone S, et al. Radiologic 
Criteria in Predicting Pathologic Less Invasive Lung Cancer According to TNM 8th Edition. Clin Lung Cancer 2019;20:163-70.

27. Kim H, Goo JM, Suh YJ, et al. Implication of total tumor size on the prognosis of patients with clinical stage IA lung adenocarcinomas appearing as part-solid nodules: Does only the solid portion size matter? Eur Radiol 2019;29:1586-94.

28. Ye T, Deng L, Wang S, et al. Lung Adenocarcinomas Manifesting as Radiological Part-Solid Nodules Define a Special Clinical Subtype. J Thorac Oncol 2019;14:617-27.

29. Miyoshi T, Aokage K, Katsumata S, et al. Ground-Glass Opacity Is a Strong Prognosticator for Pathologic Stage IA Lung Adenocarcinoma. Ann Thorac Surg 2019;108:249-55.

30. Hattori A, Hirayama S, Matsunaga T, et al. Distinct Clinicopathologic Characteristics and Prognosis Based on the Presence of Ground Glass Opacity Component in Clinical Stage IA Lung Adenocarcinoma. J Thorac Oncol 2019; 14:265-75.

31. Chiang XH, Hsu HH, Hsieh MS, et al. PropensityMatched Analysis Comparing Survival After Sublobar Resection and Lobectomy for cT1N0 Lung Adenocarcinoma. Ann Surg Oncol 2020;27:703-15.

32. Kim H, Goo JM, Kim YT, et al. Validation of the Eighth Edition Clinical T Categorization System for Clinical Stage IA, Resected Lung Adenocarcinomas: Prognostic Implications of the Ground-Glass Opacity Component. J Thorac Oncol 2020;15:580-8.

33. Akın Kabalak P, Yılmaz Ü, Ertürk H, et al. Prognostic significance of preoperative consolidation to maximum tumour diameter ratio and SUVmax in pathological stage I lung adenocarcinoma. Clin Respir J 2020;14:71-7.

34. Kuroda H, Nakada T, Oya Y, et al. Clinical adjustability of radiological tools in patients with surgically resected cT1N0-staged non-small-cell lung cancer from the longterm survival evaluation. J Thorac Dis 2020;12:6655-62.

35. Kuroda H, Nakada T, Oya Y, et al. Computed tomography and positron emission tomography-staged cN0 non-small cell lung cancer. Video-assist Thorac Surg 2020;5:14.

36. Travis WD, Brambilla E, Noguchi M, et al. International Association for the Study of Lung Cancer/American Thoracic Society/European Respiratory Society International Multidisciplinary Classification of Lung Adenocarcinoma. J Thorac Oncol 2011;6:244-85.

37. Suzuki K, Koike T, Asakawa T, et al. A prospective radiological study of thin-section computed tomography to predict pathological noninvasiveness in peripheral clinical
IA lung cancer (Japan Clinical Oncology Group 0201). J Thorac Oncol 2011;6:751-6.

38. Hayashi H, Ashizawa K, Ogihara Y, et al. Comparison between solid component size on thin-section CT and pathologic lymph node metastasis and local invasion in T1 lung adenocarcinoma. Jpn J Radiol 2017;35:109-15.

39. Sakakura N, Inaba Y, Yatabe Y, et al. Estimation of the pathological invasive size of pulmonary adenocarcinoma using high-resolution computed tomography of the chest: A consideration based on lung and mediastinal window settings. Lung Cancer 2016;95:51-6.

40. Asamura H, Hishida T, Suzuki K, et al. Radiographically determined noninvasive adenocarcinoma of the lung: survival outcomes of Japan Clinical Oncology Group 0201. J Thorac Cardiovasc Surg 2013;146:24-30.

41. Ito R, Iwano S, Kishimoto M, et al. Correlation between FDG-PET/CT findings and solid type non-small cell cancer prognostic factors: are there differences between adenocarcinoma and squamous cell carcinoma? Ann Nucl Med 2015;29:897-905.

42. Tsutani Y, Miyata Y, Misumi K, et al. Difference in prognostic significance of maximum standardized uptake value on [18F]-fluoro-2-deoxyglucose positron emission tomography between adenocarcinoma and squamous cell carcinoma of the lung. Jpn J Clin Oncol 2011;41:890-6.

43. Li M, Sun Y, Liu Y, et al. Relationship between primary lesion FDG uptake and clinical stage at PET-CT for nonsmall cell lung cancer patients: An observation. Lung Cancer 2010;68:394-7.

44. Chong S, Lee KS, Kim B, et al. Integrated PET/ CT of pulmonary neuroendocrine tumors: diagnostic and prognostic implications. AJR Am J Roentgenol 2007;188:1223-31.

45. Jackson AS, Rosenthal A, Cattoni M, et al. Staging System for Neuroendocrine Tumors of the Lung Needs to Incorporate Histologic Grade. Ann Thorac Surg 2020;109:1009-18.

46. Kuroda H, Mori S, Tanaka H, et al. Prognostic significance of combined radiologic imaging modalities for prognosis of clinical IA adenocarcinomas. Oncotarget 2017:9:10745-53.

Cite this article as: Nakada T, Kuroda H. Narrative review of optimal prognostic radiological tools using computed tomography for T1N0-staged non-small cell lung cancer. J Thorac Dis 2021;13(5):3171-3181. doi: 10.21037/jtd-20-3380 